\title{
Intermédialités
}

Histoire et théorie des arts, des lettres et des techniques

Intermediality

History and Theory of the Arts, Literature and Technologies

\section{Mitoyenneté dans le cinéma urbain de Johan van der Keuken}

\section{Marion Froger}

Numéro 14, automne 2009

bâtir

build

URI : https://id.erudit.org/iderudit/044413ar

DOI : https://doi.org/10.7202/044413ar

Aller au sommaire du numéro

Éditeur(s)

Revue Intermédialités (Presses de l’Université de Montréal)

ISSN

1705-8546 (imprimé)

1920-3136 (numérique)

Découvrir la revue

Citer cet article

Froger, M. (2009). Mitoyenneté dans le cinéma urbain de Johan van der Keuken. Intermédialités / Intermediality, (14), 127-141.

https://doi.org/10.7202/044413ar
Résumé de l'article

Qu'est-ce qu'un espace de mitoyenneté ? Ce n'est pas tout à fait un espace public, au sens où on l'entend habituellement ; c'est plutôt le lieu d'une expérience de sociabilité à la fois continuelle et précaire. Ce moment où le contact s'éprouve dans la perspective de sa rupture, cette distance entre nous qui nous fait pourtant tenir ensemble. C'est l'espace fondamental de la socialisation, pour un Isaac Joseph dont nous reprendrons les analyses afin de décrire le cinéma dit cosmopolite de Johan van der Keuken. D’Amsterdam à Groszny, Johan van der Keuken est l'un des rares cinéastes à filmer les villes en paix comme les villes en guerre, et à pouvoir ainsi montrer le coeur fragile de nos expériences sociales. Un des rares cinéastes à faire des films pour préserver l'attention au lien et promouvoir le souci de sa perte. 


\title{
Mitoyenneté dans le cinéma urbain de Johan van der Keuken
}

\author{
Marion Froger
}

\begin{abstract}
'est à Isaac Joseph que l'on doit le beau néologisme de «mitoyenneté » qu'il associe à l'idée d' « hospitalité universelle»:

si les villes peuvent peut-être nous apprendre quelque chose dans la crise actuelle du cosmopolitisme, c'est en nous rappelant qu'il n'y a pas d'ordre mondial sans une forme ou une autre d'ordre civil. [...] ce que les villes en guerre comme Beyrouth ou Sarajevo nous ont enseigné, c'est l'importance de l'espace public de circulation et sa très grande vulnérabilité puisqu'il suffit de quelques miliciens et d'un sniper pour contraindre les gens à rester chez eux. [...] L'hospitalité universelle, c'est précisément ce droit de visite sur le territoire de l'autre qui découle du simple fait de la mitoyenneté des hommes vivant sur une même planète. Avant d'être citoyens, nous sommes mitoyens et c'est dans cette proximité distante avec l'étranger que nous apprenons à donner un sens commun à la notion de monde ${ }^{1}$.
\end{abstract}

Le cinéma cosmopolite de van der Keuken a donné forme à cette idée de mitoyenneté : inlassablement, le cinéaste a circulé dans les rues des villes, sensible à cette hospitalité minimale que manifestent les gens et soucieux d'inventer la sienne d'homme à la caméra. Cinéaste mitoyen, il a eu le souci du lien faible, en cherchant l'invisible communauté des hommes hors des communautés restreintes de membres. Il l'a cherché pour lui-même dans une pratique civile du documentaire, mais aussi, puisqu'il fait des films, pour les spectateurs et les acteurs qui s'y introduisent ou les habitent. Or rien n'est moins évident que de filmer le monde comme un espace commun, et de faire d'un film un pendant imaginaire de cet espace. Van der Keuken filme la rue des villes européennes en migrant ordinaire qui a su trouver comment respecter le

1. Isaac Joseph, «Le migrant comme tout venant», dans L'athlète moral et l'enquêteur modeste [1997], Paris, Economica, 2007, p. 216. 
«droit à l'indifférence² de l'autre», avec une caméra à la main. Loin de miser sur sa discrétion et son invisibilité de caméraman, van der Keuken, au contraire, provoque l'échange tenu de regard et suppose toujours, pour lui et son spectateur, un droit de regard, qui n’est pas une indiscrétion, mais un souci du lien³. Le difficile pari de son cinéma est de produire cette proximité distante, dont parle Isaac Joseph, entre des étrangers : le filmeur, le filmé et le spectateur. Délaissant les atermoiements de la construction laborieuse de la citoyenneté européenne - à travers ses institutions lointaines et ses partages culturels plus ou moins probants -, van der Keuken a cherché, dans l'idée d'hospitalité universelle, une valeur urbaine européenne commune dont ses images portent témoignage des forces et des ruptures.

Comme le décrit avec subtilité Isaac Joseph dans Le passant considérable, nous sommes étrangers les uns aux autres dans nos pérégrinations urbaines, mais nous n'en sommes pas moins appelés tous les jours à inventer le lien de notre solidarité lâche, comme peut l'être la maille d'un chandail. Dans sa propre ville, comme dans les autres villes d'Europe ou du monde qu'il a traversées, van der Keuken vit en étranger, ou plutôt, selon la belle formule de Joseph, «en migrant tout venant» pris dans un procès infini de socialisation. La mise en scène de ce procès répond à cet impératif: reproduire l'expérience mixte de l'appartenance au tout, d'une part, et de la solitude dans la rencontre obtuse de l'autre, d'autre part.

2. Cette idée de «droit à l'indifférence de l'autre» ou d'«inattention civile», Isaac Joseph la développe à partir d'une idée présente dans les textes d'Erving Goffman, soit sous une forme active, comme tact propre à l'espace urbain de côtoiement entre étrangers, soit sous une forme passive, comme routine d'interaction permettant de préserver la sensibilité de chacun ou simplement de manifester à l'autre le respect de son quantà-soi. Il écrit par exemple dans Stigmate: «La routine des rapports sociaux dans les cadres établis nous permet d'avoir affaire aux autres, habituellement présents, sans leur accorder une attention ou des pensées particulières», Erving Goffman, Stigmates. Les usages sociaux des handicaps, trad. Alain Kihm, Paris, Les Éditions de Minuit, coll. «Le Sens commun », 1975, p. 12.

3. Dans Le passant considérable, Isaac Joseph définit l'espace public - lieu d'expérience de l'embarras, de l'ambiguïté et de la réserve dans l'interaction - comme la superposition d'un «espace-trafic » et d'un «espace-affiche» qui forment «un territoire ou une situation de co-présence», qui ouvre «la possibilité d'un échange de regard» et qui offre un «droit de regard», Isaac Joseph, Le passant considérable, Paris, Librairie des Méridiens, 1984, p. 72. 

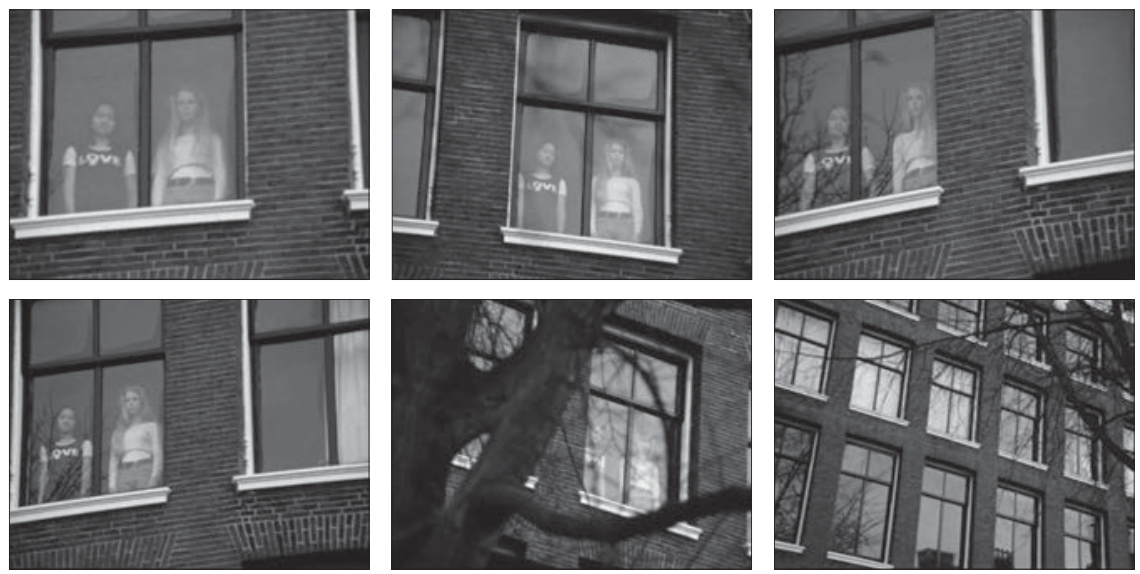

Fig. 1: Amsterdam Global Village, 1996, 229 minutes. Édition intégrale, Johan van der Keuken, vol. 2, chez Arte France Développement, Ideale Audience International, Pieter van Huystee film, 2007.

Ainsi, dans Amsterdam Global Village (1996), les longs et lents travellings pris des canaux de la ville se présentent explicitement comme des expériences de co-présence et de partage du temps. Être dans le temps, c'est s'éprouver dans et avec le monde qui s'instaure dans la perception. Le travelling ne dit pas uniquement la durée de la chose qu'il montre, mais la coexistence de ce qui existe avec celui qui le perçoit. Longueur et lenteur des travellings ont pour fonction de retarder le point de rupture qui établit la séparation du voyant et du vu. Le sentiment d'appartenance se bâtit alors sur le lien entre le corps sensible qui voit et les choses vues qui l'entourent. Mais cet abandon a pour destin d'être contrarié : l'apparition d'un visage qui vous toise affecte soudain la fluidité du mouvement. C'est ce qui arrive dans cette séquence où les travellings pris au fil de l'eau, sur les façades d'Amsterdam, sont interrompus par la présence de deux femmes à leur fenêtre, qui suivent la caméra du regard. L'échange de regards avec ces deux femmes inaccessibles dans la clôture de leur espace intime demeure indéchiffrable. Le mouvement bute sur leur apparition comme sur un écueil dans l'écoulement du temps, reprend et répète la rencontre. La série de faux raccords de mouvement qui se produit alors accentue le sentiment de rupture, exprime le hiatus qui rompt le charme de la contemplation. 

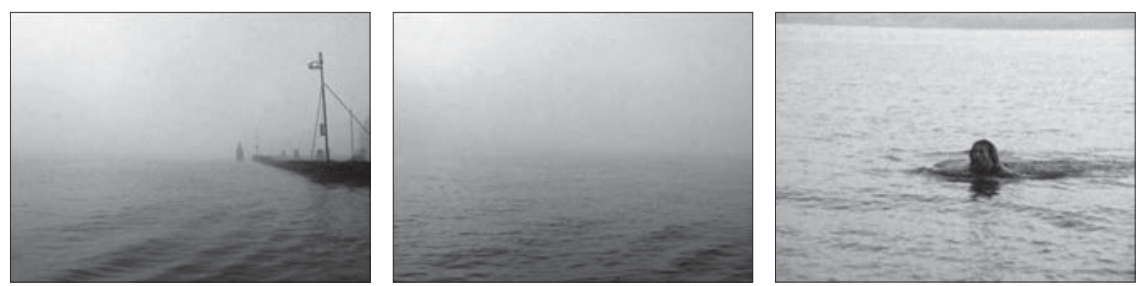

Fig. 2: Amsterdam Global Village, 1996, 229 minutes. Édition intégrale, Johan van der Keuken, vol. 2, chez Arte France Développement, Ideale Audience International, Pieter van Huystee film, 2007.

Dans une autre séquence du film, une même rencontre impromptue ${ }^{4}$ a lieu: le long travelling pris d'un bateau parcourt la rive d'un fleuve avec sa jetée, puis la surface lisse des eaux dans lesquelles le regard semblait sombrer en l'absence de tout objet. La tête d'un nageur interrompt l'abandon contemplatif au monde dans ce petit matin brumeux et silencieux où la perte de la distinction des choses renforce ce «sentiment océanique » évoqué par Romain Rolland dans une lettre à Freud ${ }^{5}$. Soudain, la présence incongrue du nageur cherchant une issue au cadre réintroduit l'expérience de la relation en dramatisant l'enjeu de réciprocité: le nageur se détourne parce qu'on le filme. Van der Keuken au cadrage ne peut se résoudre à le laisser s'éloigner. Les regards qui s'évitent, les trajectoires qui se font dévier mutuellement: tout est dit sur le fragile équilibre du lien à l'étranger, entre attention et indiscrétion, sollicitude et indifférence, aménité et agacement.

À Amsterdam, comme dans d'autres villes, le citadin doit sans cesse recomposer un lien avec de parfaits inconnus. Les échanges de regard et les modes de contact se constituent en sémiotique ténue - un tracé dévié dans le mouvement de caméra, un hiatus dans le montage -, exprimant tout à la fois la tension de l'absence de connivence et pourtant aussi le souci du lien. Ce sont là précisément les deux pôles subtils de l'espace mitoyen. La séquence de l'altercation sur les canaux d'Amsterdam lors de la fête nationale, toujours dans Amsterdam Global Village, est exemplaire à ce titre. C'est le contact brusque entre les deux canots qui déclenche l'ire du plus jeune conducteur; l'échange verbal est violent, mais

4. Même si la première, celle des deux femmes à leur fenêtre, semble être davantage le résultat d'une mise en scène.

5. La source de la religiosité serait un «Sentiment qu'il [Romain Rolland] appellerait volontiers la sensation de l'éternité, sentiment comme de quelque chose sans frontière, sans borne, pour ainsi dire "océanique" ", Sigmund Freud, Malaise dans la culture [1929], Paris, Les Presses universitaires de France, coll. «Quadrige», 1986, p. 5. 

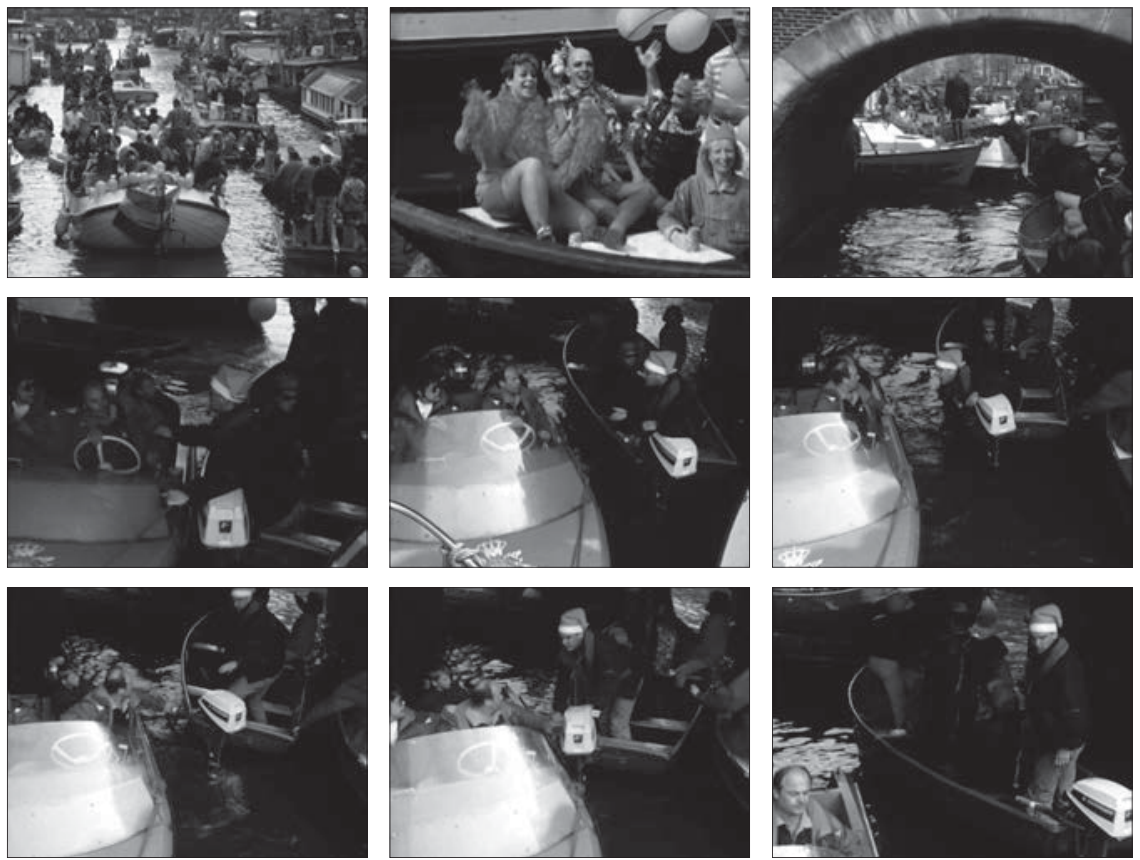

Fig. 3: Amsterdam Global Village, 1996, 229 minutes. Édition intégrale, Johan van der Keuken, vol. 2, chez Arte France Développement, Ideale Audience International, Pieter van Huystee film, 2007.

le geste presque enfantin (arroser l'autre) est tout en retenue. Les deux hommes, dont les bateaux ne s’écartent pas malgré leur désaccord, se contiennent, pour quatre raisons essentielles : l'impossibilité de fuir eut été un échange plus violent; le rythme imposé de la cohabitation lente à laquelle oblige la navigation sur le canal encombré; la présence du collectif autour d'eux, un jour précisément de célébration rituelle de la communauté; l'internalisation d'un point de vue «tiers » sur la situation qu'ils vivent et dont ils doivent sortir, qui leur permet de trouver une alternative (la pichenette d'eau) sans «perdre la face » selon l'expression bien connue de Goffman encore ${ }^{6}$. Or ces quatre raisons qui empêchent l'altercation sont à la fois extrêmement contraignantes et incroyablement fragiles. La violence (finalement) contenue de la scène et la tension qu'elle engendre rendent sensible, par la menace de sa perte, la civilité ordinaire qui fait tenir un monde commun.

6. Erving Goffman, Les rites d'interaction, trad. Alain Kihm, Paris, Les Éditions de Minuit, coll. «Le Sens commun», 1974. 
La perte du monde commun, c'est précisément ce que filme van der Keuken dans les villes en guerre qu'il a traversées. En contrepoint aux travellings paisibles pris des canaux d'Amsterdam, tout n'est, dans la séquence de Grozny, qu'éboulement, murs calcinés, fenêtres sans intérieur, enchevêtrement de ferraille et de déchets. Van der Keuken filme de l'intérieur d'une voiture les bribes de réel qui échappent constamment à sa visée du fait de l'étroitesse de l'habitacle, de la vitesse et des soubresauts chaotiques du véhicule. Le raccord heurté du mouvement de caméra se répète indéfiniment sur une ville dévastée. Le montage de la séquence amplifie ces ruptures en multipliant les interruptions de travelling sur ce qui n'a pas le temps de se dévoiler à l'image. La caméra est une intruse, les ébauches de travelling, souvent combinées à des panoramiques rapides, signifient l'hésitation du cadreur à cibler - justement - les objets. Quant aux plans fixes, très courts, ils ne laissent pas au regard le temps de se poser, dans le respect des nouvelles peurs engendrées par la guerre. L'espace de mitoyenneté a disparu et, avec lui, la chorégraphie subtile des échanges de regards et des rapports de mouvements entre les hommes et les femmes, qui craignent désormais le viseur du sniper dans l'objectif du cinéaste. Ces plans traduisent le malaise du cinéaste, qui se trouve ainsi exclu de ce qui se joue autour de lui, démuni, sans possibilité d'agir ou de compatir à la mesure de ce qui se passe. S'il filme le visage d'un enfant mort, lors de son enterrement, c'est dans le silence de sa propre sidération, le tremblé d'un gros plan obtenu par zoom, qui évoque un regard impuissant à raccorder, même dans l'après-coup du montage, ces deux réalités - la sienne et celle qu'il filme - qui composent pourtant ce temps vécu avec d'autres.

Van der Keuken n'est pas un reporter de guerre. Son cinéma est tout au contraire voué à la paix civile. Ses films urbains visent à réinventer l'espace commun composé de civilité et d'aménité qui fait la marque d'une sociabilité construite en deçà des différences cristallisées et durcies des rapports sociaux. Il s'agit plutôt de retrouver la précarité de l'expérience du lien à l'étranger, pour en ressentir la valeur et le prix. Cela se traduit, du point de vue de la forme, par des documentaires qui ne simulent pas quelque forme que ce soit de convivialité avec le spectateur, ni ne reposent sur des relations préexistantes avec les personnes filmées.

Cinéaste et cadreur, Johan van der Keuken ne déambule pas comme tout un chacun dans les rues des villes. Il construit son espace de mitoyenneté parce que la présence même de la caméra et son projet de film demandent une réinvention 

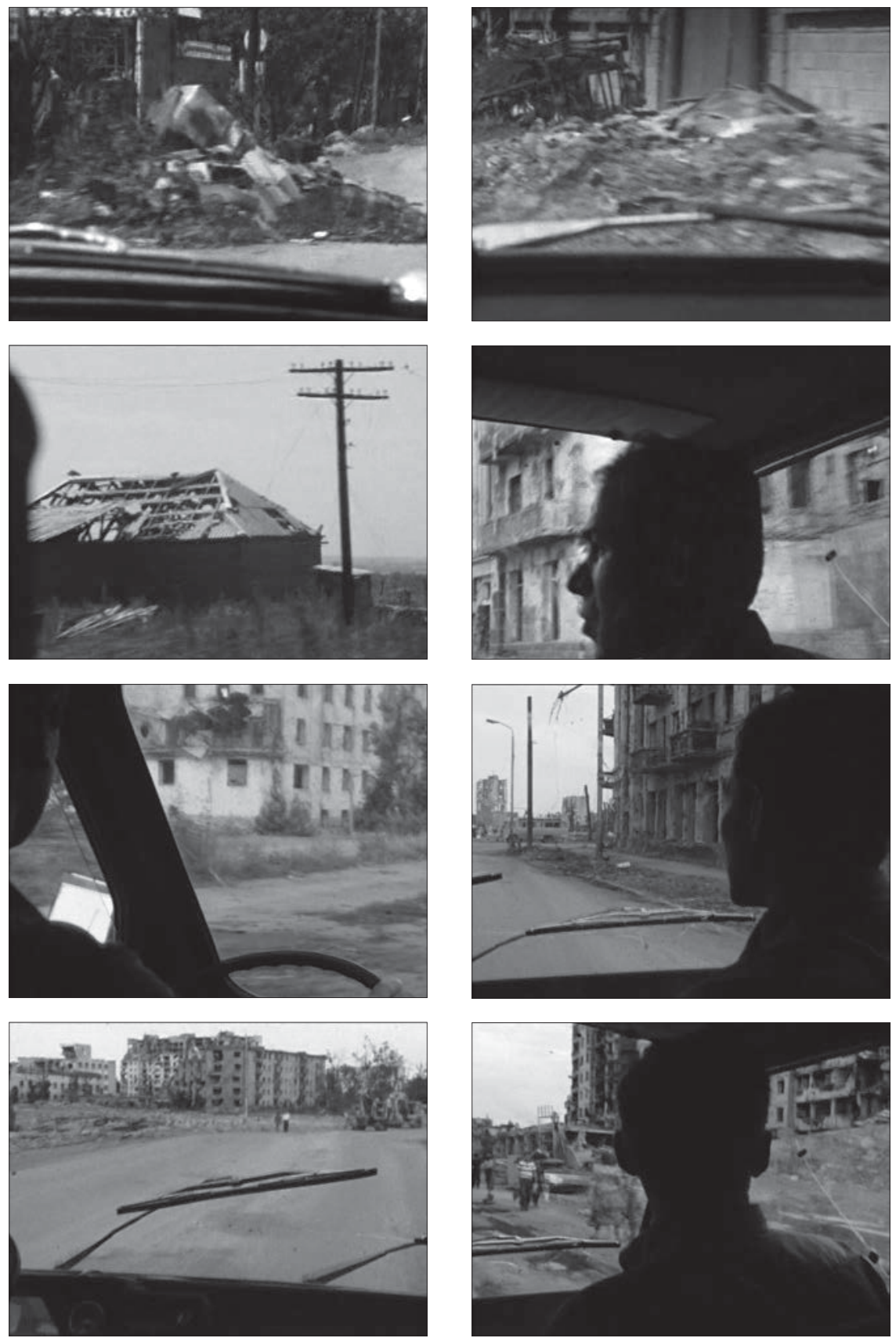

Fig. 4: Amsterdam Global Village, 1996, 229 minutes. Édition intégrale, Johan van der Keuken, vol. 2, chez Arte France Développement, Ideale Audience International, Pieter van Huystee film, 2007. 
de cet espace. Aux subterfuges techniques des documentaristes du Candid Eye et à la bonne volonté des personnes filmées du cinéma direct, qui acceptent d'oublier la caméra, van der Keuken oppose une tout autre stratégie: faire de la personne filmée l'héroïne d'un drame miniature de la rencontre. Dans Amsterdam Global Village, le cinéaste aborde un clochard d'une façon particulière: l'ayant repéré alors qu'il finissait sa nuit dans un coin de parc, il semble s'en désintéresser pour y revenir dans un jeu de cadrages qui alterne le proche et le distant, comme regarderait un passant ordinaire, sans intention précise. Il se montre curieux, mais aussi respectueux du quant-à-soi de l'homme endormi, puisqu'il détourne son attention, selon les règles tacites de civilité, vers les maçons qui refont la façade, à quelques mètres. Une manière, donc, toute cinématographique, de réinventer cette « inattention civile ${ }^{7}$ » dont Goffman parlait déjà comme le signe fort d'un lien faible. Puis le clochard s'éveille. Il demande une cigarette, que van der Keuken lui offre volontiers. Le contact est établi. L'entretien se poursuit, le courant passe assez pour que van der Keuken ose une mise en scène: il accompagne les déambulations du clochard sur quelques mètres, mais ce faisant, il détourne son objectif
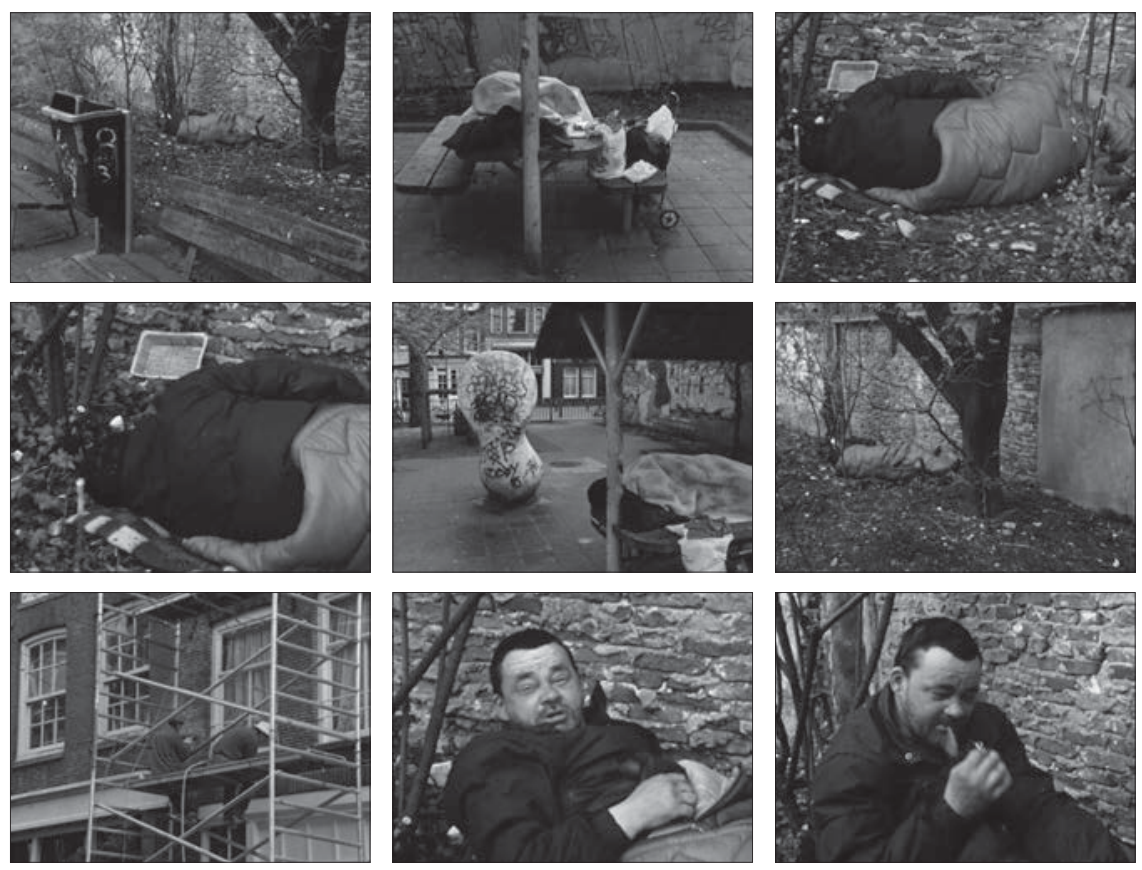

Fig. 5: Amsterdam Global Village, 1996, 229 minutes. Édition intégrale, Johan van der Keuken, vol. 2, chez Arte France Développement, Ideale Audience International, Pieter van Huystee film, 2007.

7. Voir note 2. 

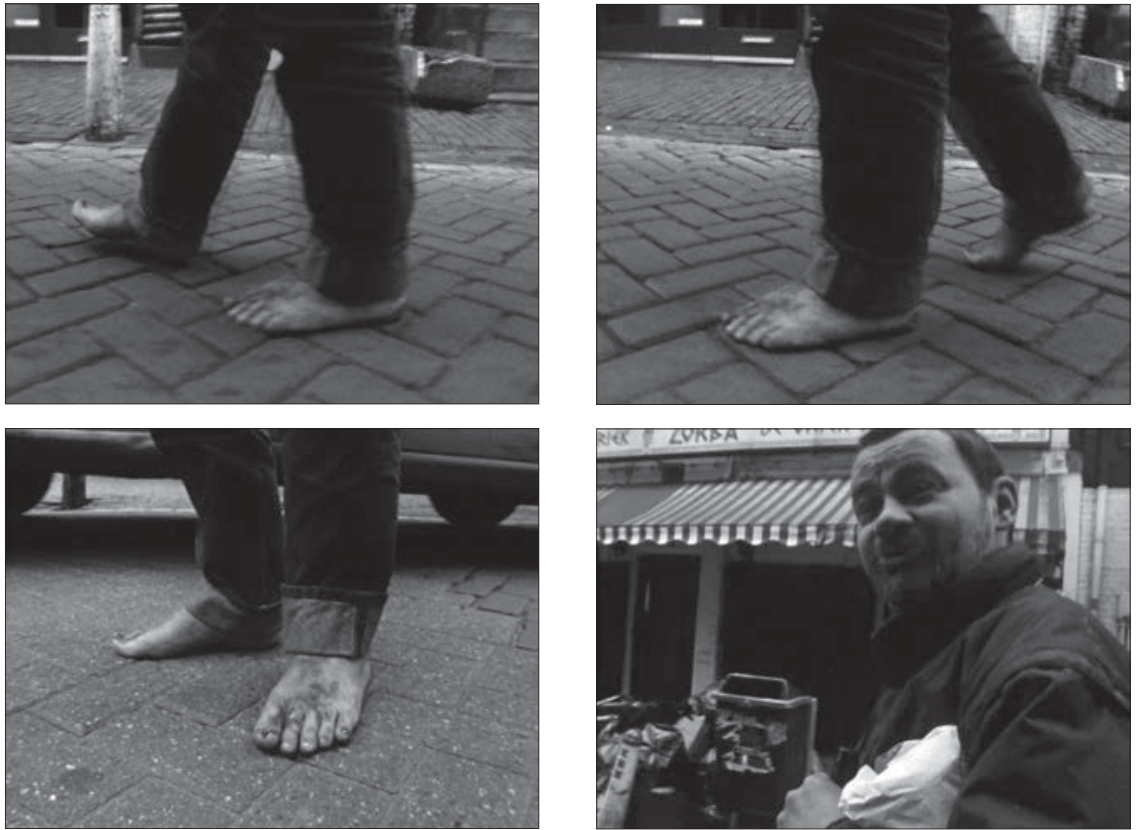

Fig. 6: Amsterdam Global Village, 1996, 229 minutes. Édition intégrale, Johan van der Keuken, vol. 2, chez Arte France Développement, Ideale Audience International, Pieter van Huystee film, 2007.

de son visage, néglige l'enregistrement de sa parole, ne cadre que ses pieds. Le montage crée une impression d’allégresse : le cinéaste semble virevolter autour du clochard et la scène se clôt sur le sourire complice de ce dernier, heureux de son personnage d'homme aux pieds nus, né d'une rencontre impromptue. L'interaction a créé une scène pour que l'exposition publique - subie plus souvent qu'autrement par les sans-abri - génère ici au contraire, en quelques plans, le plaisir partagé du spectacle entre filmeur, filmé et spectateur.

Le travail formel - mise en scène, cadrage, montage - génère des émotions sociales spécifiques, tant du côté des filmeurs/filmés que du côté des spectateurs. L'excès de mise en scène, par exemple, permet de donner corps à un espace de sociabilité propre aux films: les personnes y apparaissent plus ou moins à l'aise, se pliant plus ou moins volontiers à ce qui se trame avec elles, à la manière de ce couple qui éclate de rire dans To Sang FotoStudio (1994) à la fin de la pose que leur impose le photographe. Il n'est pas anodin de remarquer que le cinéaste a adopté, dans ce film, le modèle de création et d'interaction du photographe dont il fait le portait: Monsieur Sang met en scène chacune de ses photos avec un souci minutieux de la posture. Or van der Keuken s'y est reconnu, lui qui 

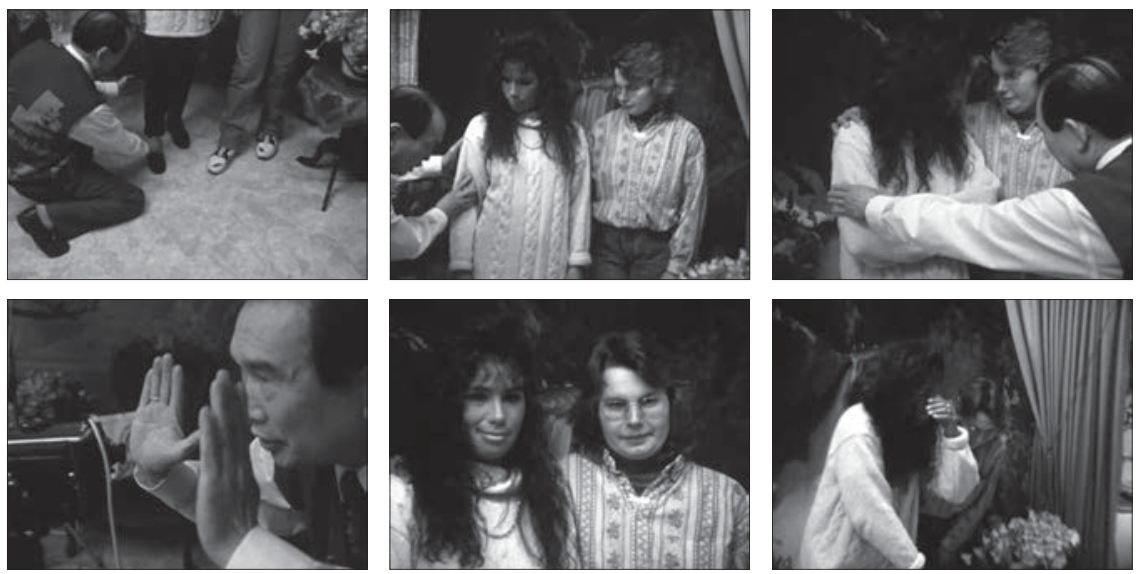

Fig 7: To Sang Fotostudio, 1997, 33 minutes. Édition intégrale, Johan van der Keuken, vol. 2, chez Arte France Développement, Ideale Audience International, Pieter van Huystee film, 2007.

demande souvent à ses modèles de répéter un geste, un mouvement, une pose, ce qu'ils font la plupart du temps de bonne grâce, même si certains lui manifestent leur agacement. Dans De beeldenstorm (La tempête d'images, 1982), le cinéaste a conservé au montage le reproche que lui adressait son modèle; dans Amsterdam Global Village, il met en scène le refus du coursier de continuer le film - qui se termine alors avec lui. To Sang FotoStudio permet au cinéaste d'aller au bout de la logique de son travail formel - chacun n'est qu'une forme dans l'image - sans perdre de vue la sociabilité que ce travail implique. Et c'est certainement l'aspect réflexif de ce film qui lui inspira l'idée d'un making of (Leven met je ogen, 1997) dont l'auteur (Ramon Gieling) ne se fit pas prier non plus pour diriger le maître documentariste comme un acteur en lui imposant la «pose» devant la caméra. Cette mise en abîme du geste de création reposant sur l'exposition d'autrui à la caméra nous renvoie à la fois aux accords tacites et miraculeux de l'abandon de la personne filmée, et aux gênes, aux ridicules, aux exaspérations qu'entraîne le travail formel durant l'interaction de la prise de vue.

Mais le projet du cinéaste n'est pas non plus toujours tout à fait transparent pour les personnes qu'il filme. «Au revoir, petite forme », dit-il sur les dernières images d'Hermann, l'enfant aveugle dont il vient de faire le portrait (Blind Kind, 1964), signalant au passage le hiatus entre l'expérience relationnelle vive et le projet artistique dont cet enfant, privé de vue, ne pourra pas prendre la mesure. C'est pourquoi le spectateur, dès lors qu'il épouse le point de vue de l'enfant comme personne filmée privée de son image, hésite, n'adhère que partiellement 


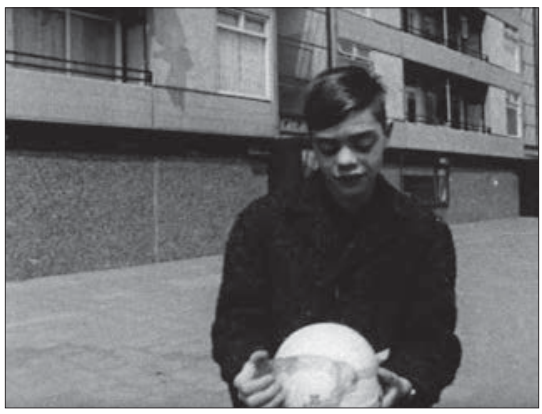

Fig. 8: L'enfant aveugle, 1964, 24 minutes. Édition intégrale, Johan van der Keuken, vol. 3, chez Arte France Développement, Ideale Audience International, Pieter van Huystee film, 2007.

à cette entreprise formelle à laquelle l'enfant n'a pas accès. Le commentaire en voix hors champ de van der Keuken sur l'image d'Hermann atteste de la balance inégale du pouvoir de signifier. Du coup, certaines questions surgissent: quand une personne se laisse filmer, à qui et à quoi cède-t-elle ainsi? L'expérience de voir un tel film se fait épreuve de sociabilité à son tour dans les ambiguïtés de la démarche du cinéaste; dans les obscurités des motivations des personnes filmées. On éprouve les tensions de la sociabilité entre mondanité et tact, cabotinage et pudeur, calcul et générosité, capture de l'autre et «captation mutuelle ${ }^{8}$ ». Il s'agit donc de vivre un jeu subtil de rapports sur un terrain imaginaire - le cinéma comme espace transitionnel -, un jeu de sociabilité aiguisant la sensibilité pour préserver l'attention au lien et promouvoir le souci de sa perte.

Loin de disparaître pour obtenir la pâte lisse d'un discours documentaire, le malaise de la non-réciprocité est toujours présent dans les contacts liminaires entre étrangers - ce que nous demeurons après la vision d'un film. C'est en

8. Dans un article intitulé «résistance et sociabilités», Isaac Joseph évoque, après Simmel, le moment fort de la sociabilité, celui qui se détache du quotidien. Il s'agit d'un abandon non calculé qui crée, entre des sujets, un lien très fort alors même que chacun conserve son opacité. La confiance se vit comme un état, plutôt que comme un résultat: d'où le caractère fortuit de ces moments "parce qu'on y va toujours, en quelque sorte, à la pêche à la ligne. Il arrive que notre pari soit le bon. C'est donc un processus de captation, plus que de fascination ou d'identification réciproque. Et précisément parce que ce que l'on capte, on en ignore la source, l'organisation en amont, la ligne de pente, ces moments sont très fragiles. » Isaac Joseph, « résistance et sociabilités », dans L'athlète moral et l'enquêteur modeste, Paris, Économica, 2007, p. 130. 


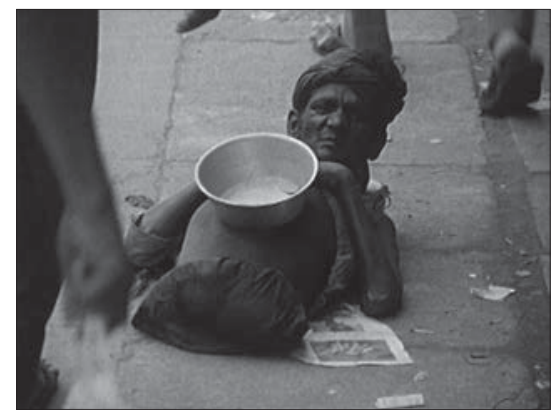

Fig 9: L'œil au-dessus du puits, 1988, 90 minutes. Édition intégrale, Johan van der Keuken, vol. 1, chez Arte France Développement, Ideale Audience International, Pieter van Huystee film, 2007.

tremblant, nous dit van der Keuken, qu'il a filmé cet hindou infirme dans les rues de New Delhi, avec la conscience contradictoire de devoir le faire - pour son film (Het oog boven de put, 1988) - tout en se jugeant impardonnable de l'avoir fait'. «Les interactions dites mixtes sont [donc] typiquement flottantes et angoissées, agressives et embarrassées ${ }^{10}$. Van der Keuken, sans en rajouter dans la violence de l'interaction, touche ici aux limites du partage, touche à ce moment critique où tout peut basculer entre des «mitoyens» parce que les conditions d'existence sont inégales; parce que la vulnérabilité ne vous lie plus, mais vous oppose; parce qu'il y a de l'intraitable dans tout rapport de soi à l'autre. Il touche ici au franchissement qui fait que les atrocités deviennent soudainement possibles, déjà faites ou à venir. Ce n'est donc pas pour rien qu'il se rend à Sarajevo ou à Grozny avec cette question en tête: que leur est-il arrivé? Et cette piste de réponse : savons-nous vivre ensemble en étrangers? C'est précisément cette question que le petit film Sarajevo Film Festival Film (1994) met en scène dans sa séquence d'ouverture. Les longs plans fixes sur les visages, qui ont exigé des personnes filmées qu'elles prennent la pose, révèlent alors toute leur pertinence: ils n'incitent pas au déchiffrement de visages qui restent impassibles, mais initient à l'accompagnement de l'étranger, dont on sent vibrer la conscience sans forcément pénétrer ni l'âme ni les sentiments. Or c'est cet accompagnement, ce côtoiement et cette mitoyenneté qui précisément font défaut à Sarajevo.

9. Johan van der Keuken : «Je l'ai approché vraiment en tremblant... Je l'ai regardé, il m’a regardé... et c'est ça... c'est le moment d'abîme dans le film, le moment impardonnable.»

10. Joseph, 2007, p. 219. Isaac Joseph paraphrase ici Erving Goffman: «les situations sociales mixtes tendent à produire des interactions flottantes et angoissées », dans Goffman, 1975 , p. 30. 

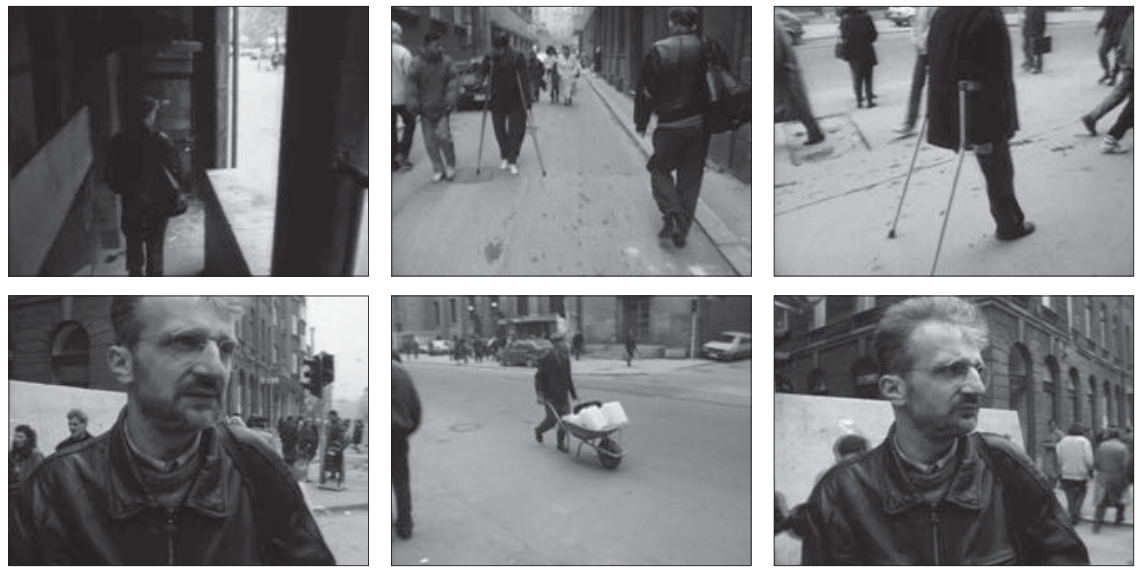

Fig. 10: Sarajevo Film Festival Film, 1994, 14 minutes. Édition intégrale, Johan van der Keuken, vol. 2, chez

Arte France Développement, Ideale Audience International, Pieter van Huystee film, 2007

Au tout début de Sarajevo Film Festival Film, on emboîte le pas à un inconnu, à travers des couloirs sombres. Arrivé dans la rue, l'inconnu s'arrête, la contemple en silence tandis qu'on - cinéaste, spectateur, passant - le dévisage. Mais une voix hors champ, la sienne, s'insinue et raconte le quotidien de la ville, la peur de la déambulation, la méfiance des uns envers les autres. Van der Keuken ose alors des allers-retours interrogatifs entre le personnage immobile au milieu de la rue qui paraît songer à la perte du commun, dont nous entretient sa voix en son hors champ, et les passants affairés, qui transportent eau et nourriture avec une diligence inquiète. Larrêt du personnage, les allers-retours indécis de la caméra, et l'écoute de cette voix intérieure, font de ce moment de côtoiement et de partage de l'espace civil une sorte de miracle, tel que peut encore l'offrir le cinéma - comme pratique d'interaction tout autant que comme espace imaginaire de sociabilitée ${ }^{11}$ - à cette ville en guerre.

11. Le film se poursuit d'ailleurs autour du témoignage d'une spectatrice assidue du Festival du film de Sarajevo qui explique qu'elle brave les dangers de la rue, le sniper embusqué destructeur de l'espace mitoyen qu'évoquait Isaac Joseph dans la citation qui ouvre cet article, pour aller vivre ce qui lui semble désormais le bien le plus précieux: une expérience sociale pacifiée avec les personnages des films. 


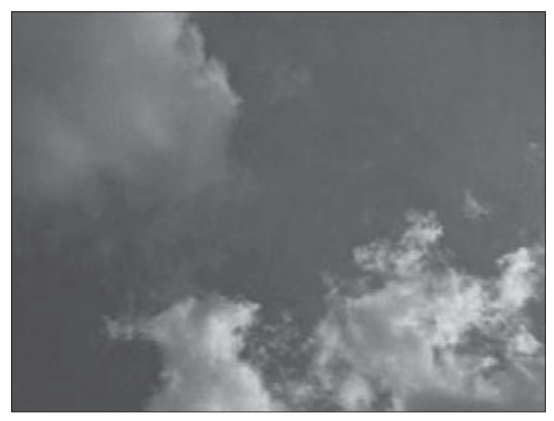

Figure. 11

140

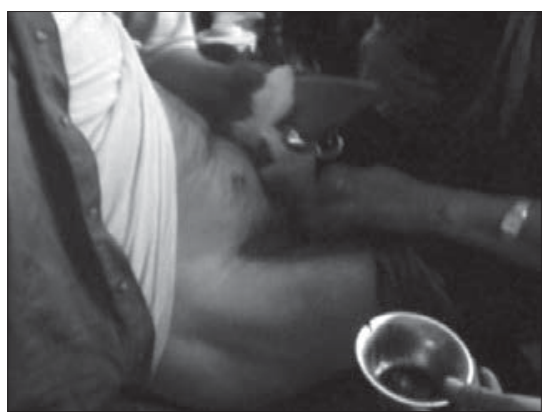

Figure. 13

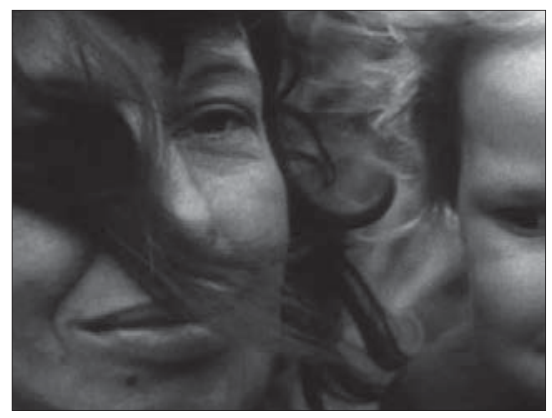

Figure. 12

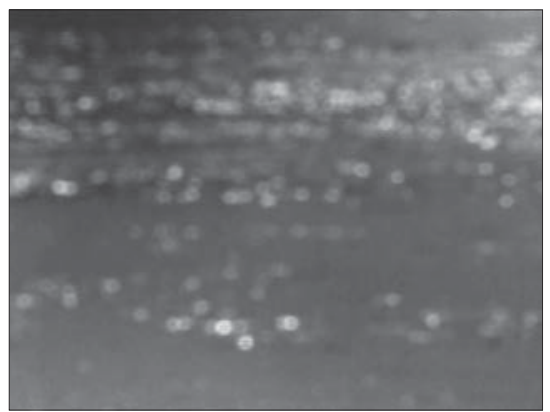

Figure. 14

Fig. 11, 12, 13 et 14: Vacances prolongées, 2000, 142 minutes. Édition intégrale, Johan van der Keuken, vol. 5, chez Arte France Développement, Ideale Audience International, Pieter van Huystee film, 2007.

L'Europe n'existe pas, elle ne fait que survivre aux films de van der Keuken comme un souvenir de rencontres, de cohabitations éphémères, de liens flottants. Elle a eu lieu, dans des films qui coupent court à la réalité géographique, tout en créant sans cesse des espaces où coexister. Les films de van der Keuken ne cèdent en rien au cinéma des identités. Il fait plutôt un cinéma des sociabilités infimes et considérables, pour reprendre les mots d'Isaac Joseph.

Dans De Grote Vakantie (Vacances prolongées, 2000), le film que van der Keuken entreprend alors qu'il apprend qu'il va bientôt mourir, les travellings laissent les choses entrer et sortir du cadre, pour poursuivre leur destin en dehors: une manière pour van der Keuken d'appréhender la mort annoncée. Il ne construit plus rien avec d'autres, il aborde un dernier voyage qu'il doit faire seul; il n'est plus dans le travail de socialisation de ce «migrant tout venant» qu'il a choisi d'incarner comme posture de cinéaste. Il est dans l'inventaire des choses dont il lui faut se déprendre: un ciel de nuages, un visage aimé, un corps céleste 
de cinéaste (car c'est un corps souffrant qu'il montre au spectateur), jusqu'au contour même des choses.

«Je n'ai pas de style », dit en substance van der Keuken devant des étudiants de la FEMIS, dans le documentaire que Thierry Nouel lui consacre (Johan van der Keuken, 1997). Parce que son souci, ce n'est pas le style, en effet, mais la forme; la forme d'une expérience de mitoyenneté; celle de l'équilibre et du basculement; du lointain et du proche. Mais avec le risque du formalisme qui fait perdre de vue cette expérience du commun que l'on n'emporte pas dans la mort, mais qu'on laisse en héritage aux vivants. 